\title{
The Management of Diabetic Foot Ulcers with Porcine Small Intestine Submucosa Tri-Layer Matrix: A Randomized Controlled Trial
}

\author{
Shawn M. Cazzell, ${ }^{1-3}$ Darrell L. Lange, \\ Jaime E. Dickerson, Jr. ${ }^{4,5} *$ and Herbert B. Slade ${ }^{4,6}$ \\ ${ }^{1}$ Valley Vascular Surgery Associates of Fresno, Fresno, California. \\ ${ }^{2}$ Limb Preservation Platform, Inc., Fresno, California. \\ ${ }^{3}$ Division of Podiatry, Department of Surgery, University of California, San Francisco, San Francisco, California. \\ ${ }^{4}$ Smith and Nephew, Inc., Fort Worth, Texas. \\ Departments of ${ }^{5} /$ mmunology and Cell Biology and ${ }^{6}$ Pediatrics, University of North Texas Health Science Center, \\ Fort Worth, Texas.
}

Objective: This study demonstrates that superior outcomes are possible when diabetic foot ulcers (DFU) are managed with tri-layer porcine small intestine submucosa (SIS).

Approach: Patients with DFU from 11 centers participated in this prospective randomized controlled trial. Qualified subjects were randomized (1:1) to either SIS or standard care (SC) selected at the discretion of the Investigator and followed for 12 weeks or complete ulcer closure.

Results: Eighty-two subjects (41 in each group) were evaluable in the intentto-treat analysis. Ulcers managed with SIS had a significantly greater proportion closed by 12 weeks than for the Control group (54\% vs. 32\%, $p=0.021$ ) and this proportion was numerically higher at all visits. Time to closure for ulcers achieving closure was 2 weeks earlier for the SIS group than for SC. Median reduction in ulcer area was significantly greater for SIS at each weekly visit (all $p$ values $<0.05$ ). Review of reported adverse events found no safety concerns.

Innovation: These data support the use of tri-layer SIS for the effective management of DFU.

Conclusion: In this randomized controlled trial, SIS was found to be associated with more rapid improvement, and a higher likelihood of achieving complete ulcer closure than those ulcers treated with SC.

\section{INTRODUCTION}

THE PREVALENCE of diabetes mellitus in the United States is currently estimated to be $9.3 \%$ of the total population (29.1 million affected people). The prevalence is even higher within special populations for example, some minority groups or the elderly. ${ }^{1} \mathrm{Al}-$ though the annual increases observed over the previous two decades may be leveling off, ${ }^{2}$ diabetes and its downstream consequences remain a serious and costly public health problem. Diabetic foot ulcers (DFU) are a frequent and serious complication of diabetes mellitus with an annual incidence rate of $1 \%-4 \%$ and a lifetime risk of $15 \%-25 \% .^{3-5}$ DFU are often

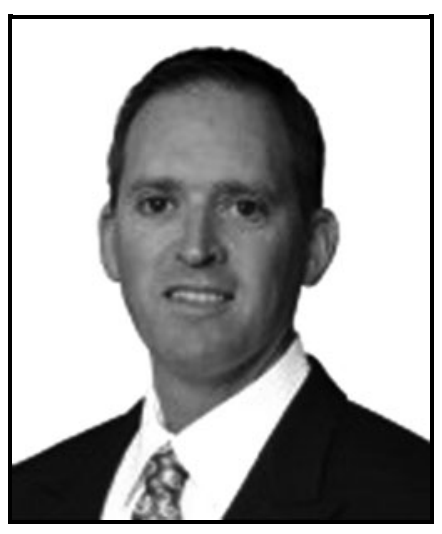

Shawn M. Cazzell, DPM

Submitted for publication February 20, 2015. Accepted in revised form March 3, 2015.

*Correspondence: Medical and Clinical Affairs, Smith and Nephew, Inc., 3909 Hulen Street, Fort Worth, TX 76107 (e-mail: jaime.dickerson@smith-nephew.com). 
difficult to heal, and may become chronic, substantially increasing the risk that they become infected leading to hospital admissions and, too frequently, lower limb amputations. ${ }^{6}$

The goal of DFU management is to promote rapid and complete reepithelialization to minimize the risk of ulcer complications and to restore the patient's quality of life to a "pre-ulcer" status. Successful management of DFU must address several deficits inherent to these ulcers. These factors may include any or all of the following: poor blood glucose control, necrotic tissue, infection, poor or no off-loading, chronic inflammation, and inadequate perfusion. Many ulcers fail to make progress toward healing despite the best efforts made to address these contributing problems. Good standard care (SC), that is, debridement of necrotic tissue, infection control, off-loading, and maintenance of a moist wound environment has been reported to result in the healing of about one-fourth to one-half of DFU by 20 weeks. ${ }^{7,8}$ Some gains have been made through the use of approaches such as "living skin equivalents" or "dermal substitutes," for example, Marston; ${ }^{9}$ however, the cost of these therapies can be a barrier to widespread use and the incremental benefit above SC have generally been modest. Clearly, an alternative method of ulcer management that leads to superior outcomes and at a reasonable cost would be highly desirable.

One such alternative is tri-layer porcine small intestine submucosa (SIS) (OASIS $^{\circledR}$ Ultra; Cook Biotech, Inc., West Lafayette, IN; exclusively marketed by Smith and Nephew, Inc., Fort Worth, TX). SIS is a three-dimensional biomaterial $\sim 0.30 \mathrm{~mm}$ thick consisting of a biocompatible, acellular, collagen-based (predominantly types I, III, and V) extracellular matrix (ECM). The material possesses several advantages including relative low cost, long shelf life, storage at room temperature, low risk of immunological reaction, and the retention of the natural ECM architecture. ${ }^{10}$ Single-layer porcine SIS has been successfully used for the management of many types of cutaneous wounds including venous/arterial ulcers and DFU. ${ }^{11,12}$ A reasonable hypothesis for the efficacy of the product is that the three-dimensional natural architecture provides an appropriate environment for infiltrating cells, ${ }^{10}$ however, it is also likely that the SIS composition, including collagen, proteoglycans, and glycoproteins, closely mimics normal skin ECM function. ${ }^{13,14}$

Tri-layer SIS has not been previously evaluated in a randomized controlled trial. The purpose of this study was to demonstrate that superior outcomes are possible when DFU are managed with tri-layer SIS. The results presented below provide support for this objective.

\section{CLINICAL PROBLEM ADDRESSED}

DFU pose several challenges for the practitioner. Contributing factors will very likely remain ongoing as various therapies are employed in the effort to heal. It is critically important for the wellbeing of the patient not only to heal these ulcers, but to heal them rapidly to avoid the pernicious sequelae of amputation and, in some cases, death. This trial demonstrates that management of these ulcers with SIS can provide outcomes significantly better than obtained with usual SC in terms of both closure and time to closure.

\section{MATERIALS AND METHODS}

\section{Study participants}

Eligible patients were 18 years or older with a diagnosis of type 1 or 2 diabetes mellitus requiring medications to control blood glucose levels. Subjects were required to have a neuropathic foot ulcer on the plantar surface $>6$-week and $\leq 1$ year duration between 0.5 and $10 \mathrm{~cm}^{2}$ in area. Additional requirements were adequate perfusion to the affected foot, no clinical signs or symptoms of ulcer infection, serum albumin $\geq 2.0 \mathrm{~g} / \mathrm{dL}$, serum prealbumin $\geq 10 \mathrm{mg} / \mathrm{dL}$, and $\mathrm{HbA} 1 \mathrm{c} \leq 12 \%$.

\section{Ethics statement}

This study was performed in compliance with the ethical principles of the Declaration of Helsinki and Good Clinical Practice. The study protocol, investigators, and consent documents were reviewed and approved by the Institutional Review Board (IRB, Sterling IRB, Atlanta, GA), and all patients provided written informed consent before taking part in the study. This study was registered at ClinicalTrials.gov as NCT01835379 (http:/ clinicaltrials.gov/ct2/show/NCT01835379).

\section{Study design}

This was a randomized, parallel group, openlabel, multicenter, 16-week clinical study (including a 12-week treatment period, Figs. 1 and 2) designed to compare clinical outcomes for DFU managed with tri-layer porcine SIS, or with SC as selected by the Investigator. The study was carried out at 11 sites in California, Indiana, Louisiana, Texas, and Virginia. The study was conducted between May 2013 and July 2014. Randomization to treatment was centralized using a computer-generated sequence with treatment assignment made by the electronic data capture system at the randomization visit. Subjects were thus randomized to 


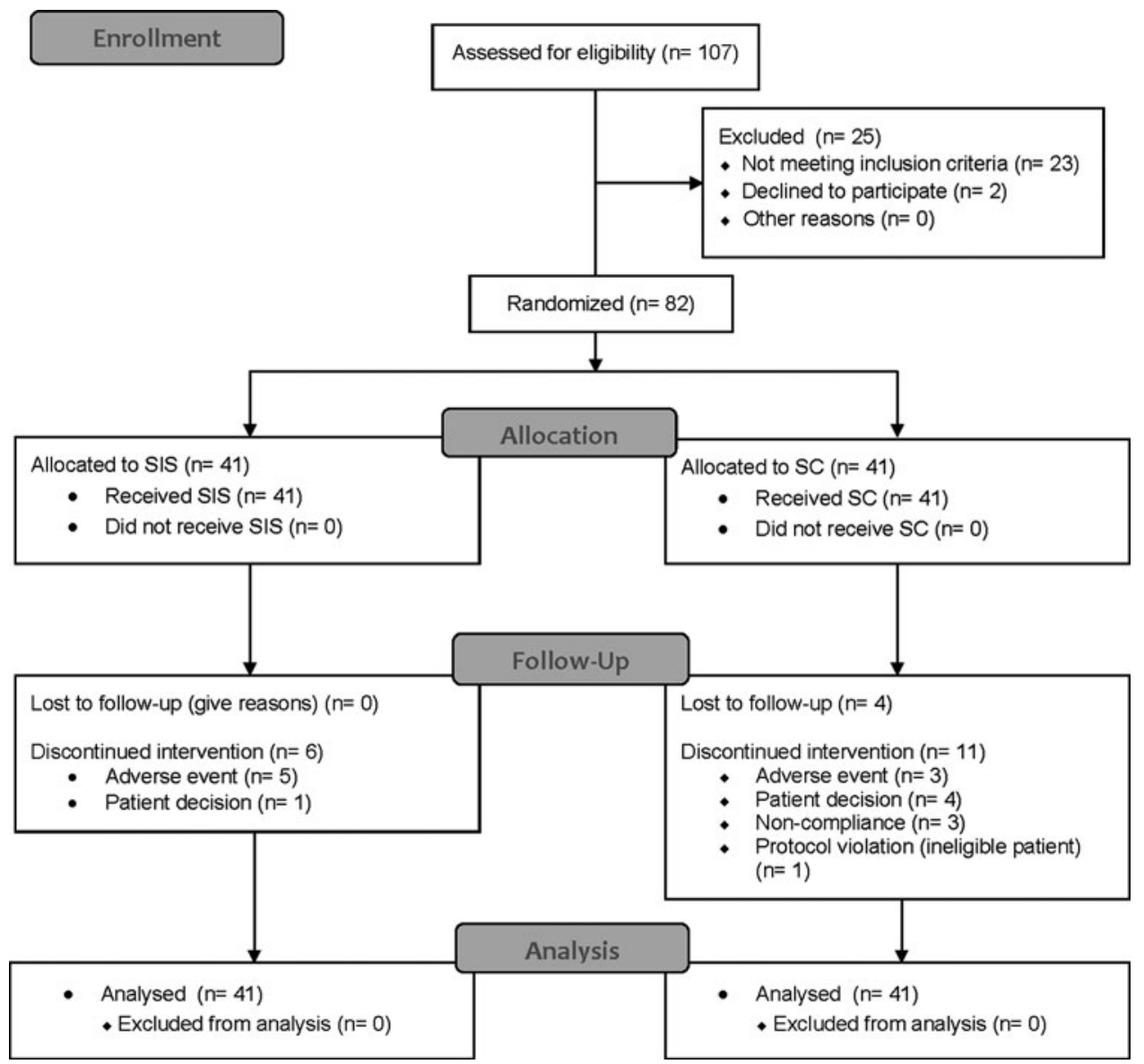

Figure 1. CONSORT flow diagram. SIS, tri-layer porcine small intestine submucosa. Standard care, standard care as selected by Investigator. All patients randomized were included in the intent-to-treat data set, which was used for primary inference.

treatment in the order they qualified across all study sites. Randomization was stratified by wound size $\left(0.5-2.0 \mathrm{~cm}^{2}\right.$, and $\left.>2.0 \mathrm{~cm}^{2}\right)$ to prevent dissimilar ulcer size distributions between the two treatment groups. The trial was open-label because of obvious dissimilarities between SIS and the various SC treatments.

Patients were evaluated for eligibility at a screening visit that included assessment of hematology and blood chemistry. Basic demographic

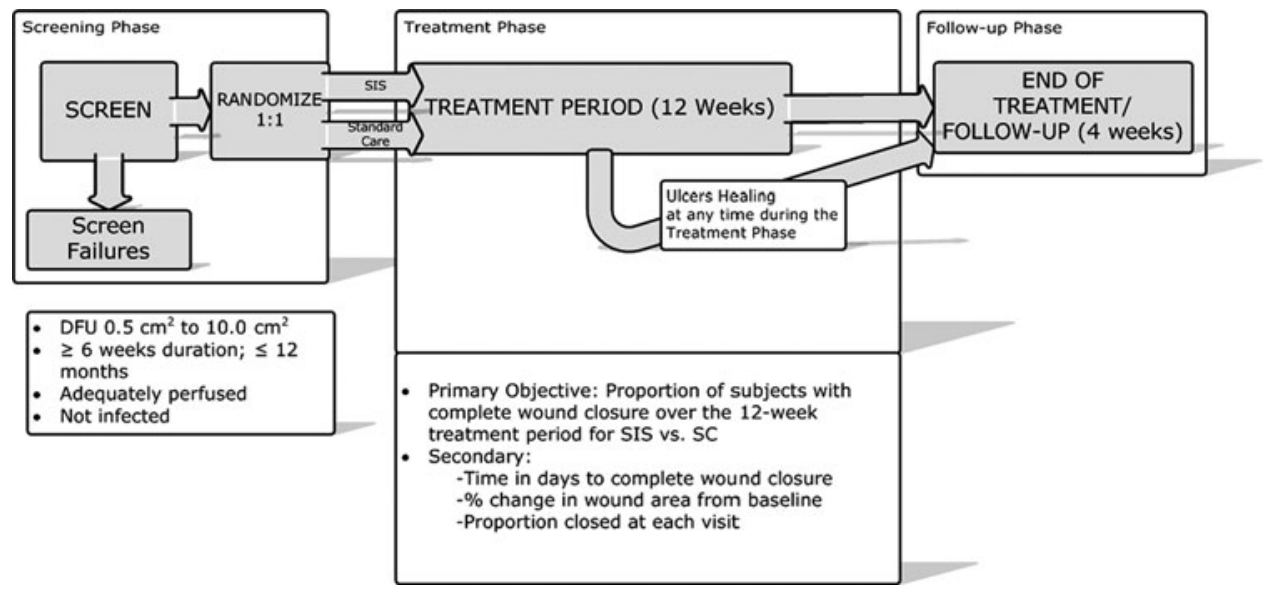

Figure 2. Study schematic. SIS, tri-layer porcine small intestine submucosa. Standard care, standard care as selected by Investigator. 
information included self-reported race, ethnicity, and gender. Eligible patients reported for the baseline/randomization visit from 1 to 8 days later. Ulcers were surgically debrided and callus was removed as appropriate followed by baseline wound area measurement. All patients were provided with an offloading device (Darco, Huntington, WV) that they were required to wear. Patients were then randomly assigned to treatment in an equal allocation ratio to either the SIS group or to the control group.

Patients returned weekly for 12 weeks for ulcer assessment/measurement, recording of any adverse events (AE) that may have occurred, debridement (for SC patients only, sharp debridement was not allowed in the SIS group), and application of SIS or the assigned SC. At the end of 12 weeks, or at any other visit for a patient achieving closure, patients entered a follow-up period returning after an additional 4 weeks. For ulcers not closed during the treatment period, there were no restrictions on permissible ulcer treatments. The follow-up period was designed to assess durability of closures achieved during the treatment period, record additional closures, and to follow any ongoing AE.

SIS was applied once each week to ulcers following cleansing with sterile saline. The matrix was cut so that there was an approximate 1/8 inch overlap on the wound edge, secured with adhesive tape (Steri-Strips ${ }^{\mathrm{TM}}$ ), moistened with saline, and covered with a non-adherent dressing (Mepitel ${ }^{\mathrm{TM}}$ ), a foam dressing (Allevyn ${ }^{\mathrm{TM}}$ ), and maintained in place with a self-adherent wrap bandage (Coban $^{\mathrm{TM}}$ ). Steri-Strips ${ }^{\mathrm{TM}}$ and Coban ${ }^{\mathrm{TM}}$ are products of 3M Co (St. Paul, MN). Mepitel ${ }^{\mathrm{TM}}$ is a product of Mölnlycke Healthcare (Gothenburg, Sweden). Allevyn $^{\mathrm{TM}}$ is a product of Smith and Nephew, Inc. (Hull, United Kingdom). Patients randomized to the control group received wound care/dressings as deemed appropriate by the Investigator with the exception of enzymatic debridement, growth factors, skin equivalents or dermal substitutes, topical antibiotics, and hyperbaric or negative pressure therapies. A listing of the care provided in the control group is given in Fig. 3 .

\section{Sample size}

Based on published data for the single-layer matrix product, ${ }^{11,12}$ it was assumed that there would be a $30 \%$ difference in proportion of closed ulcers at 12 weeks between SIS and SC. A sample size of 49 subjects per group was planned to provide a statistical power of $80 \%$ with $\alpha=0.05$ (two-sided). The sample size estimate was revised downward to

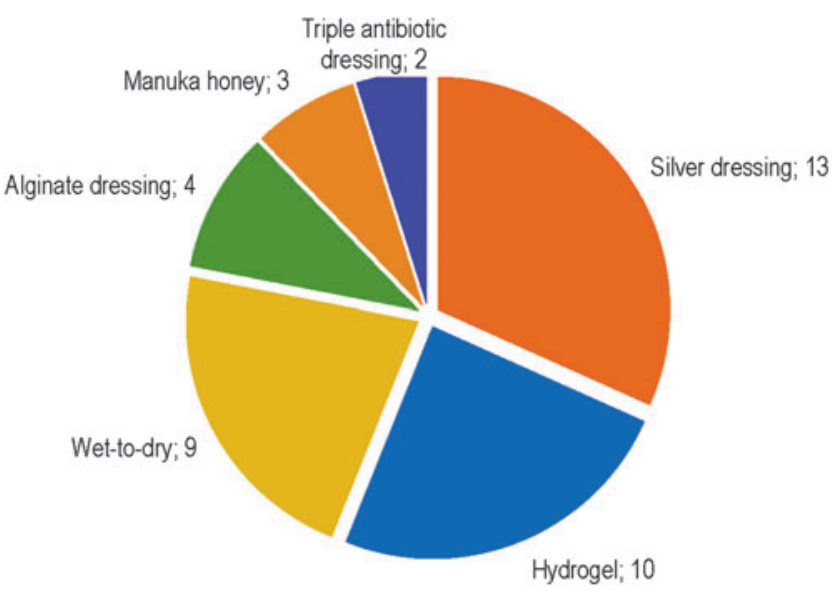

Figure 3. Standard care selected by the Investigator; numerals indicate number of patients treated with various standard care choices.

40 subjects per group following a preplanned interim analysis when the first 40 subjects enrolled had completed treatment.

\section{Endpoints}

The primary endpoint was the proportion of subjects with complete ulcer closure over the 12-week treatment period. Secondary efficacy variables included time in days to complete ulcer closure over the treatment period, percent change in ulcer area from baseline for each visit, proportion of subjects with complete ulcer closure at each visit, and durability of closure/additional new closures recorded at the 16-week follow-up visit.

\section{Study assessments}

Ulcer area was measured at each study visit using the ARANZ Silhouette ${ }^{\mathrm{TM}}$ digital image capture and wound measurement device (ARANZ Medical, Christchurch, New Zealand). Ulcer closure was defined as $100 \%$ reepithelialization, no drainage, and no need for a dressing. Neuropathy was confirmed at screening by subject inability to perceive $10 \mathrm{~g}$ pressure using a nylon monofilament on five plantar areas of the foot: great toe, the first, third, and fifth metatarsal heads, and the fourth digit. A subject's foot was considered neuropathic if at least three of the tested areas had no sensation. Adequate perfusion to the affected foot was confirmed by ankle/brachial index $>0.7$, or alternatively, a transcutaneous oxygen pressure $>40 \mathrm{~mm}$ $\mathrm{Hg}$, a great toe pressure of $>40 \mathrm{~mm} \mathrm{Hg}$, or a Doppler waveform consistent with adequate blood flow.

Safety was assessed through analysis of $\mathrm{AE}$, both volunteered and elicited. AE were collected from the date a subject provided informed consent through follow-up and exit from the study. 


\section{Statistical analysis}

SAS $^{\circledR}$ Version 9.0 was utilized to perform the statistical analysis. Hypothesis testing was performed at the 5\% significance level. The intentto-treat (ITT) population, defined as all of the randomized subjects, was used for primary inference. Proportion of subjects with complete ulcer closure over the 12-week treatment period was evaluated using the non-parametric CochranMantel-Haenszel (CMH) test with adjustment for site. The primary endpoint was also analyzed for the per protocol (PP) population and for race, age, and gender subgroups; results for PP and these subgroups were found to be similar to the ITT results presented below.

Time in days to complete ulcer closure over the treatment period was analyzed using the Cox regression/proportional hazards model. Treatment was included as the main predictor, whereas subject baseline wound size and duration were included as covariates. Percent change in ulcer area from baseline for each visit was compared for between-group differences using a two-way analysis of covariance (ANCOVA). The ANCOVA model included treatment group, pooled site, and baseline ulcer size as covariates. The CMH test was used to examine treatment effects on the proportion of subjects with complete ulcer closure at each visit and to evaluate durability of closure/additional new closures recorded at the 16-week follow-up visit (4 weeks post-treatment).

The overall treatment effect on proportion of ulcers closed over the entire 12 weeks and the interaction of treatment by week was evaluated using the general estimation equation model. ${ }^{15}$

Missing data due to either missed visits or because of ulcer closure or exit from the study before week 12 were imputed by the method of last observation carried forward.

\section{RESULTS}

A total of 82 patients provided informed consent and were randomized to treatment; 41 in each treatment group. All 82 patients were considered evaluable for the ITT analysis. Table 1 summarizes the demographic and wound characteristics for the study population. The majority of subjects were $<65$ years of age $(78 \%)$, male $(76 \%)$, white $(81 \%)$, and non-Hispanic/Latino (68\%). Study wounds were generally small with a median area of $1.1 \mathrm{~cm}^{2}$ (mean area $=2.3 \mathrm{~cm}^{2}$ ) and a median duration of 18 weeks (mean duration $=21.8$ weeks). There were no significant differences between treatment groups for any of these parameters.
Table 1. Demographics and baseline wound characteristics

\begin{tabular}{|c|c|c|c|c|}
\hline \multirow[b]{2}{*}{ Demographic } & \multirow[b]{2}{*}{ Total (82) } & \multicolumn{2}{|c|}{ Treatment group } & \multirow{2}{*}{$\begin{array}{c}\text { p-value } \\
\text { (ANOVA) } \\
\text { Chi-square }\end{array}$} \\
\hline & & $S I S(n=41)$ & $S C(n=41)$ & \\
\hline \multicolumn{5}{|l|}{ Age } \\
\hline Mean & 56.9 & 57.1 & 56.6 & \multirow[t]{6}{*}{0.8318} \\
\hline Median & 56.0 & 56.0 & 56.0 & \\
\hline Standard deviation & 10.8 & 10.9 & 10.8 & \\
\hline Min-Max & 23-85 & 23-85 & 34-80 & \\
\hline$<65$ years & $64(78 \%)$ & $33(81 \%)$ & $31(76 \%)$ & \\
\hline$\geq 65$ years & $18(22 \%)$ & $8(20 \%)$ & $10(24 \%)$ & \\
\hline \multicolumn{5}{|l|}{ Gender } \\
\hline Female & $20(24 \%)$ & $9(22 \%)$ & $11(27 \%)$ & \multirow[t]{2}{*}{0.6070} \\
\hline Male & $62(76 \%)$ & $32(78 \%)$ & $30(73 \%)$ & \\
\hline \multicolumn{5}{|l|}{ Race } \\
\hline Non-White & $16(20 \%)$ & $8(20 \%)$ & $8(20 \%)$ & \multirow[t]{2}{*}{0.7212} \\
\hline White & $66(81 \%)$ & $33(81 \%)$ & $33(81 \%)$ & \\
\hline \multicolumn{5}{|l|}{ Ethnicity } \\
\hline Hispanic/Latino & $26(32 \%)$ & $10(24 \%)$ & $16(39 \%)$ & \multirow[t]{2}{*}{0.1545} \\
\hline Not Hispanic/Latino & $56(68 \%)$ & $31(76 \%)$ & $25(61 \%)$ & \\
\hline \multicolumn{5}{|l|}{ Wound area $\left(\mathrm{cm}^{2}\right)$} \\
\hline Mean & 2.3 & 2.1 & 2.6 & \multirow[t]{4}{*}{0.6495} \\
\hline Median & 1.1 & 1.2 & 1.0 & \\
\hline Standard deviation & 5.5 & 2.3 & 7.5 & \\
\hline Min-Max ${ }^{\mathrm{a}}$ & $0.3-48.4$ & $0.3-10.5$ & $0.4-48.4$ & \\
\hline \multicolumn{5}{|l|}{ Ulcer duration (weeks) } \\
\hline Mean & 21.8 & 21.3 & 22.2 & \multirow[t]{4}{*}{0.7716} \\
\hline Median & 18.0 & 19.0 & 18.0 & \\
\hline Standard deviation & 12.8 & 12.3 & 13.5 & \\
\hline Min-Max & $7.0-49.0$ & $7.0-49.0$ & $7.0-49.0$ & \\
\hline
\end{tabular}

${ }^{\mathrm{a}}$ Min and Max exceed the inclusion range $\left(0.5-10 \mathrm{~cm}^{2}\right)$ because of ulcer changes between screening visit and subject randomization.

SIS, tri-layer small intestine submucosa; SC, standard care as selected by the Investigator; ANOVA, analysis of variance.

\section{Proportion of patients with closed ulcers at 12 weeks}

A significantly greater proportion of ulcers were closed by week 12 for the SIS group than for the SC group (54\%, $N=22$ vs. $32 \%, N=13 ; p=0.021$ ), a difference of $22 \%$ (Fig. 4). Similar differences between the groups in proportion closed were consistently observed in the subgroup analyses; males, $27 \%$ greater for SIS $(p=0.024)$, females, $20 \%$, Caucasian patients, $22 \%$ ( $p=0.042)$, non-Caucasian patients, $25 \%$, patients 50 years of age or older, $15 \%$, and patients $<50$ years of age, $40 \%$.

\section{Time in days to complete ulcer closure}

Cox regression analysis demonstrated that treatment group assignment was the most important predictor of closure over the treatment period with a hazard ratio of $2.005(p=0.049)$. Ulcer duration was found to have a modest, but significant, negative association with closure (hazard ratio $0.964, p=0.018$ ). Kaplan-Meier survivor analysis shows that the probability of closure at week 12 was $62 \%$ for the SIS group compared with $40 \%$ for SC. Median time to closure was 12 weeks for SIS. 


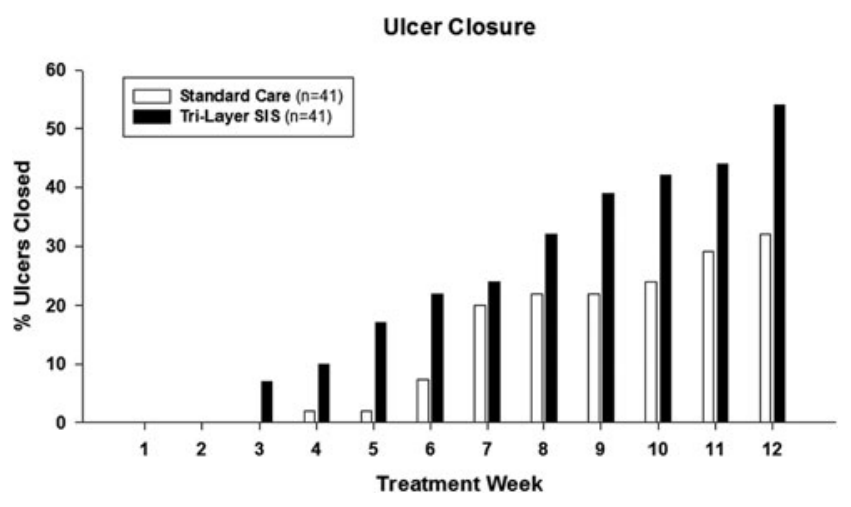

Figure 4. Proportion of closed ulcers at each visit. Tri-layer SIS, tri-layer porcine small intestine submucosa; standard care, standard care as selected by Investigator.

Median time to closure could not be calculated for SC as less than half had closed by the end of treatment. Considering only those ulcers that did close, median time to closure was 9 weeks for SIS versus 11 weeks for SC.

\section{Percent change in ulcer area}

Median percent change (reduction) in ulcer area for each visit was greater for SIS than for SC at all treatment visits (all $p$ values $<0.05$, Fig. 5) beginning as early as 1 week post-baseline (SIS-34\%, SC-15\%) and continuing through to week 12 (SIS-100\%, SC-70\%).

\section{Proportion of ulcers closed at each visit}

There were more ulcers closed at each study visit for SIS than for SC beginning at week 3 when the first three ulcers had closed, all in the SIS group

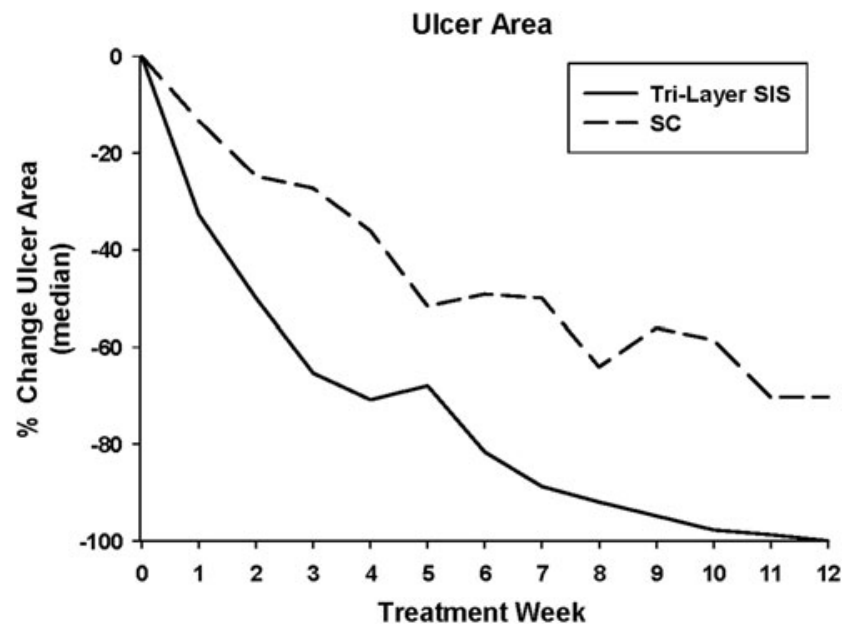

Figure 5. Median percent change in ulcer area from baseline. Tri-layer SIS, tri-layer porcine small intestine submucosa; standard care, standard care as selected by Investigator.
(Fig. 4). The difference in proportion closed was statistically significant at two of the visits, week 5 ( $p=0.027)$ and week $12(p=0.021)$. Overall treatment effect on proportion closed over the entire 12 weeks and the interaction of treatment by week was also found to be statistically significant $(p=0.047)$ in favor of the SIS group.

\section{Durability of closure and additional closures}

Patients completing the treatment period returned after an additional 4 weeks to assess durability of closure for ulcers that had closed by week 12 , and to record new closures that may have occurred. The number of ulcers closed at the 16 -week follow-up visit remained unchanged for both the SIS and $\mathrm{SC}$ groups ( $\mathrm{SIS}=22, \mathrm{SC}=13$ ), however, there were ulcer recurrences and new closures for both groups. Five ulcers reopened in the SIS group while there were five new closures. One ulcer reopened in the SC group with one new closure recorded.

\section{Adverse events}

$\mathrm{AE}$ were obtained as solicited comments from study patients and as observations by the Investigator. Similar to studies of pharmaceutical agents, $\mathrm{AE}$ were defined as any untoward change (expected or unexpected) in a subject's medical health that occurred after informed consent had been obtained. AE were distributed evenly across both treatment groups with 26 patients in each group experiencing $\mathrm{AE}$ (63\%, each group). All of these $\mathrm{AE}$ are common to the patient population under study and only one, for "maceration" of the periwound area was considered to be related to study treatment (SIS) by the Investigator. This AE was mild in severity and was resolved $\sim 1$ week after cessation of treatment.

\section{DISCUSSION}

DFU are difficult to heal and may often become chronic or "stalled." The microenvironment of the wound bed for a chronic or stalled ulcer is very different from that of an acute or healing ulcer, typically with high levels of inflammatory mediators and proteases. ${ }^{16,17}$ The ECM is of key importance in orchestrating epithelialization and angiogenesis of the healing wound. ${ }^{13}$ In addition to contributing to tissue structural integrity and strength, the matrix provides a framework for ingrowth of vascular endothelial cells and providing an appropriate substrate for keratinocyte migration and attachment. ${ }^{10}$ Proteoglycans of the matrix are important in the maintenance of water balance. ECM proteins also bind and sequester growth fac- 
tors, functioning as a depot. ${ }^{14}$ Given the importance of the ECM it is not surprising that nonhealing diabetic ulcers have a generally aberrant ECM in which the component proteins have been modified by glycation, oxidation, and proteolytic attack from endogenous matrix metalloproteinases. ${ }^{13,18}$ There is also a deficit in the formation of new ECM in DFU due to both diminished synthesis and an increased rate of removal resulting from the highly proteolytic environment. ${ }^{19,20}$

It is an accepted tenet that for a wound to close inflammation must subside and healthy granulation tissue must be formed. ${ }^{21}$ It may be that application of an ECM material such as SIS helps to address this imbalance between formation and destruction of ECM in the wound bed. SIS may tip the balance away from the proinflammatory, proteolytic status quo of the chronic wound to an environment more supportive of vascular ingrowth and, ultimately, epithelium formation. The clinical data of this study are consistent with this hypothesis. As early as 1 week following randomization, there was a significant difference in the percent reduction in ulcer area for the ulcers managed with SIS and those managed with SC (33\% vs. $13 \%$, $p=0.017$ ) while closure was first achieved for three ulcers at week 3, all in the "SIS managed" group. Indeed, the group managed with SIS had consistently better outcomes throughout the study in terms of proportion closed, reduction in size, and time to closure. The populations randomized to either SIS or to SC were not found to significantly differ in any of the baseline demographic or ulcer characteristics measured. Therefore, it is not unreasonable to conclude that it was the different management methods employed that led to these disparate outcomes. This is not to say that wounds managed with passive SC methods primarily aimed at maintenance of a moist wound environment will not close; in fact $32 \%$ did achieve closure by week 12 and although this percentage was unchanged at week 16, given sufficient time additional closures may have occurred. The important point is that as long as a DFU remains open, it is at risk of becoming infected and the patient is at risk of complications that may be as serious as loss of a limb and even death. Patients whose DFU were managed with SIS were, on average, more rapidly closed, and more likely to close than their counterparts managed with SC.

A limitation of this study is the unblinded design. This was chosen intentionally to allow clinically relevant $\mathrm{SC}$ in the control group. Treatment allocation bias was controlled through randomization and bias in outcomes through the use of ob-

\section{KEY FINDINGS}

- More DFU were closed with tri-layer SIS than with SC chosen by the Investigators.

- Of the ulcers that closed, the median time to closure was 2 weeks earlier for ulcers managed with tri-layer SIS.

- The chance of DFU closure was two times greater when tri-layer SIS was applied compared to SC.

jective endpoints (e.g., closure) or measurements made with a digital wound measurement device (ARANZ Medical). Post-treatment follow-up was limited to 4 weeks, which may not be of sufficient length to fairly evaluate durability of closure. However, this, in our view, affects both groups equally. The authors are unaware of any biological differences in reepithelialization attributable to the management regimen and therefore do not feel that the short follow-up provides an advantage to either treatment group. The diversity of SC therapies allowed in the control group could be considered a weakness; however, the fact is that there is not a single accepted SC treatment consistently employed even among those considered expert in the wound-healing field. Allowing the study Investigators to choose the SC they believed to be most appropriate was, in our view, the most relevant and "real-world" control group possible.

This study provides Level I evidence ${ }^{22}$ regarding the outcomes that can be achieved in the management of DFU with tri-layer SIS, outcomes found to be significantly better than those associated with investigator-selected SC. More DFU managed with SIS achieved closure, and did so more rapidly than ulcers managed with SC. Faster, more predictable closure is surely the aim of all wound care practitioners.

\section{INNOVATION}

DFU are among the most difficult wounds to heal and are a substantial challenge for the wound care practitioner. Tri-layer SIS is a novel product indicated for the management of various types of wounds, including DFU. This clinical trial provides Level I evidence of the effective management of DFU with tri-layer SIS; DFU were over twice as likely to close when managed with SIS as compared to SC.

\section{ACKNOWLEDGMENTS AND FUNDING SOURCES}

This research study was sponsored by Smith and Nephew. 


\section{AUTHOR DISCLOSURE AND GHOSTWRITING}

Dr. S.M.C. was a principal investigator on this clinical study funded by Smith and Nephew. Drs. J.E.D., D.L.L., and H.B.S. are employees of Smith and Nephew. No ghostwriter was used for the preparation of this article.

\section{ABOUT THE AUTHORS}

Shawn M. Cazzell, DPM is a practicing podiatrist in Fresno, CA at Valley Vascular Surgery Associates. He is also the Chief Medical Officer, Division of Chronic Wound and Podiatric Research, for Limb Preservation Platform, Inc., a freestanding wound and clinical research facility dedicated to chronic wound management, vascular pathology, and related conditions. Dr. Cazzell also has an appointment in the Department of Surgery, Division of Podiatry at the University of California, San Francisco, CA. He has participated in multiple clinical research studies as a principal investigator. Darrell L. Lange, $\mathbf{P h D}$ is a Senior Manager in Medical and Clinical Affairs at Smith and Nephew. He was the study manager for this study. Jaime E. Dickerson, $\mathbf{P h D}$ is the Vice President of Medical and Clinical Affairs at Smith and Nephew. $\mathrm{He}$ is also Adjunct Associate Professor in the Department of Immunology and Cell Biology at the University of North Texas Health Science Center, Fort Worth, TX. Herbert B. Slade, MD is the Chief Scientific Officer and Chief Medical Officer for Smith and Nephew. He also has an appointment with the Department of Pediatrics at the University of North Texas Health Science Center in Fort Worth, TX.

\section{REFERENCES}

1. Centers for Disease Control and Prevention. National Diabetes Statistics Report: estimates of diabetes and its burden in the United States 2014. 2014. Atlanta, GA: U.S. Department of Health and Human Services. http://cdc.gov/ diabetes/pubs/statsreport14/national-diabetesreport-web.pdf (last accessed October 19, 2014).

2. Geiss LS, Wang J, Cheng YJ. Prevalence and incidence trends for diagnosed diabetes among adults aged 20 to 79 years, United States, 19802012. JAMA 2014;312:1218-1226.

3. Singh N, Armstrong DG, Lipsky BA. Preventing foot ulcers in patients with diabetes. JAMA 2005 293:217-228

4. Boulton AJM, Kirsner RS, Vileikyte L. Neuropathic diabetic foot ulcers. N Engl J Med 2004;351: 48-55.

5. Lavery LA, Armstrong DG, Wunderlich RP, Tredwell J, Boulton AJM. Diabetic foot syndrome: evaluating the prevalence and incidence of foot pathology in Mexican Americans and non-hispanic whites from a diabetes disease management cohort. Diabetes Care 2003;26:1435-1438.

6. Alavi A, Sibbald RG, Mayer D, et al. Diabetic foot ulcers. Part II. Management. J Am Acad Derm 2014;70:21.e1-e24.

7. Margolis DJ, Kantor J, Berlin JA. Healing of diabetic neuropathic foot ulcers receiving standard treatment. A meta-analysis. Diabetes Care 1999; 22:692-695.

8. Margolis DJ, Allen-Taylor L, Hoffstad O, Berlin JA. Healing diabetic neuropathic foot ulcers: are we getting better? Diabet Med 2005;22:172-176.
9. Marston WA. The efficacy and safety of Dermagraft in improving the healing of chronic diabetic foot ulcers: results of a prospective randomized trial. Diabetes Care 2003;26:1701-1705.

10. Brown-Etris M, Cutshall WC, Hiles MC. A new biomaterial derived from small intestine submucosa and developed into a wound matrix device. Wounds 2002;14:150-166.

11. Romanelli M. Randomized comparison of OASIS wound matrix versus moist wound dressing in the treatment of difficult-to-heal wounds of mixed arterial/venous etiology. Adv Skin Wound Care 2010;23:34-38.

12. Niezgoda JA, Van Gils CC, Frykberg RG, Hodde JP. Randomized clinical trial comparing OASIS wound matrix to Regranex gel for diabetic ulcers. Adv Skin Wound Care 2005;18:258-266.

13. Davidson JM, DiPietro L. The wound healing process. In: Veves A, Giurini JM, LoGerfo FW, eds. The Diabetic Foot. Totowa, NJ: Humana Press, 2006:59-82.

14. Roberts D. Emerging functions of matricellular proteins. Cell Mol Life Sci 2011;68:3133-3136.

15. Zeger SL, Liang KY. An overview of methods for the analysis of longitudinal data. Stat Med 1992;11:1825-1839

16. Brem H, Stojadinovic O, Diegelmann RF, et al. Molecular markers in patients with chronic wounds to guide surgical debridement. Mol Med 2007;13:30-39.

17. Galperin RC, Lange DL, Ramsay SJ, et al. Antiinflammatory effects of clostridial collagenase: results from in vitro and clinical studies. JAPMA 2015;105.
18. Lev-Tov H, Li CS, Dahle S, Isseroff RR. Cellular versus acellular matrix devices in treatment of diabetic foot ulcers: study protocol for a comparative efficacy randomized controlled trial. Trials 2013; 14:8.

19. Blakytny R, Jude EB. Altered molecular mechanisms of diabetic foot ulcers. Int J Low Extrem Wounds 2009;8:95-104.

20. Lobmann R, Schultz G, Lehnert H. Proteases and the diabetic foot syndrome: mechanisms and therapeutic implications. Diabetes Care 2005;28: 461-471.

21. Holloway S, Harding K, Stechmiller JK, Schultz G. Acute and chronic wound healing. In: Baranoski $S$, Ayello EA, eds. Wound Care Essentials. Ambler, PA: Lippincott Williams and Wilkins, 2012:83-100.

22. Sackett DL. Rules of evidence and clinical recommendations on the use of antithrombotic agents. Chest 1989;95:2S-4S.

\section{Abbreviations and Acronyms \\ $\mathrm{AE}=$ adverse events \\ ANCOVA $=$ analysis of covariance \\ ANOVA $=$ analysis of variance \\ $\mathrm{CMH}=$ Cochran-Mantel-Haenszel test \\ $\mathrm{DFU}=$ diabetic foot ulcer \\ $\mathrm{ECM}=$ extracellular matrix \\ $\mathrm{IRB}=$ institutional review board \\ $\mathrm{ITT}=$ intent-to-treat \\ $\mathrm{PP}=$ per protocol \\ $\mathrm{SC}=$ standard care \\ SIS = small intestine submucosa}

